\title{
WHO IS WHO IN ALGOL-LAND ? \\ - PART I -
}

\author{
J.P. DE GREVE and W. PACKET \\ Astrophysical Institute, V.U.B. \\ Pleinlaan 2 \\ B 1050 Brussels \\ Belgium \\ (Not reviewed)
}

\begin{abstract}
We computed the evolution through case A mass transfer for 8 systems with mass of the primary equal to 3 and $5 \mathrm{M}_{\mathrm{o}}$, mass ratios 0.7 and 0.9 , and different periods. To this we added similar results from Packet (1988) for $\mathrm{M}_{\mathrm{i}}=9 \mathrm{M}_{\mathrm{o}}, \mathrm{q}_{\mathrm{i}}=0.6$, $\mathrm{P}_{\mathrm{i}}=1.62 \mathrm{~d}$.

During the mass transfer two competing mechanisms in the gainer decide on the evolution of the system: the rejuvenation of this star as the increasing convective core mixes fresh hydrogen into the inner regions, and the acceleration of nuclear burning, responding to the increasing mass.

In all the cases the net result is a faster decrease of the central hydrogen content compared to the mass losing star. The secondary fills its own critical Roche lobe and reversed mass transfer starts.

From our results and those of Nakamura and Nakamura (1984), we find that reversed mass transfer occurs after core hydrogen burning of the secondary (case $\mathrm{A}_{1} \mathrm{~B}_{2}$ ) approximately for periods larger than $1 \mathrm{~d}\left(\mathrm{M}_{1 \mathrm{i}}=3 \mathrm{M}_{\mathrm{O}}\right)$ to $2 \mathrm{~d}\left(\mathrm{M}_{1 \mathrm{i}}=13.4 \mathrm{M}_{\mathrm{O}}\right)$. For smaller periods this happens before the gainer ends its core hydrogen burning (case $\left.\mathrm{A}_{1} \mathrm{~A}_{2}\right)$.

Packet, W.: 1988, Ph. D. thesis, V.U.B., Brussels.

Nakamura, M., Nakamura, Y.: 1984, Astrophys. Space Sci. 104, 367.
\end{abstract}

Space Science Reviews 50 (1989), 338.

(C) 1989 by Kluwer Academic Publishers. Printed in Belgium. 